\title{
Modified Mesoporous Silica Nanoparticles with a Dual Synergetic Antibacterial Effect
}

DOI:

10.1021/acsami.7b14642

\section{Document Version}

Accepted author manuscript

Link to publication record in Manchester Research Explorer

\section{Citation for published version (APA):}

Michailidis, M., Sorzabal-Bellido, I., Adamidou, E. A., Diaz-Fernandez, Y. A., Aveyard, J., Wengier, R., Grigoriev, D., Raval, R., Benayahu, Y., D'Sa, R. A., \& Shchukin, D. (2017). Modified Mesoporous Silica Nanoparticles with a Dual Synergetic Antibacterial Effect. ACS Applied Materials and Interfaces, 9(44), 38364-38372.

https://doi.org/10.1021/acsami.7b14642

\section{Published in:}

ACS Applied Materials and Interfaces

\section{Citing this paper}

Please note that where the full-text provided on Manchester Research Explorer is the Author Accepted Manuscript or Proof version this may differ from the final Published version. If citing, it is advised that you check and use the publisher's definitive version.

\section{General rights}

Copyright and moral rights for the publications made accessible in the Research Explorer are retained by the authors and/or other copyright owners and it is a condition of accessing publications that users recognise and abide by the legal requirements associated with these rights.

\section{Takedown policy}

If you believe that this document breaches copyright please refer to the University of Manchester's Takedown Procedures [http://man.ac.uk/04Y6Bo] or contact uml.scholarlycommunications@manchester.ac.uk providing relevant details, so we can investigate your claim.

\section{OPEN ACCESS}




\section{Modified Mesoporous Silica Nanoparticles with}

\section{Dual Synergetic Antibacterial Effect}

Marios Michailidis ${ }^{* \dagger}$, Ioritz Sorzabal-Bellido ${ }^{\ddagger}$ Evanthia A. Adamidou ${ }^{\S}$, Yuri Antonio Diaz-

Fernandez ${ }^{\ddagger}$, Jenny Aveyard", Reut Wengier ${ }^{\perp}$, Dmitry Grigoriev ${ }^{\natural}$, Rasmita Raval ${ }^{\ddagger}$, Yehuda Benayahu $^{\perp}$, Raechelle A. D’Sall, Dmitry Shchukin ${ }^{*, \dagger}$

${ }^{\dagger}$ Stephenson Institute for Renewable Energy, Department of Chemistry, University of Liverpool, Crown Street, Liverpool L69 7ZD, UK

${ }^{\ddagger}$ Open Innovation Hub for Antimicrobial Surfaces at the Surface Science Research Centre, University of Liverpool, Oxford Street, L69 3BX, Liverpool, UK.

${ }^{\S}$ Manchester Institute of Biotechnology, School of Chemical Engineering and Analytical

Science, The University of Manchester,131 Princess Street, M1 7DN, Manchester, UK.

" Department of Mechanical, Materials and Aerospace Engineering, University of Liverpool, L69 3GH, UK.

『 Fraunhofer Institute for Applied Polymer Research IAP Functional Protein Systems/Biotechnology, Geiselbergstrasse 69, 14476 Potsdam-Golm, Germany.

${ }^{\perp}$ School of Zoology, George S. Wise Faculty of Life Sciences, Tel Aviv University, Ramat Aviv, Tel Aviv 69978, Israel. 
KEYWORDS: colloids, silica, surface modification, porous materials, nanoparticles, quaternary ammonium salts, encapsulation.

ABSTRACT. Application of mesoporous silica nanoparticles as antifouling/antibacterial carriers is limited and specifically with dual synergetic effect. In the present work, mesoporous silica nanoparticles (MSNs) modified with quaternary ammonium salts (QASs) and loaded with the biocide Parmetol S15 were synthesised as functional fillers for antifouling/antibacterial coatings. From the family of the mesoporous silica nanoparticles, MCM-48 was selected as a carrier due to its cubic pore structure, high surface area and high specific pore volume. The quaternary ammonium salts used for the surface modification of MCM-48 were dimethyloctadecyl [3(trimethoxysilyl) propyl] ammonium chloride and dimethyltetradecyl [3-(triethoxysilyl) propyl] ammonium chloride. The QAS-modified MCM-48 reveal strong covalent bonds between the QAS and the surface of the nanoparticles. The surface functionalization was confirmed by Fourier transform infrared spectroscopy (FTIR), thermogravimetric analysis (TGA), elemental analysis and $\zeta$-potential measurements. Additional loading of the QAS-modified MCM-48 with a commercially available biocide (Parmetol S15) resulted in synergetic dual antibacterial/antifouling effect. Either loaded or unloaded QAS-modified MSNs exhibited high antibacterial performance confirming their dual activity. The QAS-modified MCM-48 loaded with the biocide Parmetol S15 killed all the exposed bacteria after 3 hours incubation and presented $100 \%$ reduction at the antibacterial tests against Gram negative and Gram positive bacteria. Furthermore, the QAS-modified MCM-48 without Parmetol S15 presented 77-89\% reduction against the exposed Gram negative bacteria and $78-94 \%$ reduction against the exposed Gram positive bacteria. In addition, the modified MCM-48 were mixed with coating formulations and their antifouling performance was assessed in a field test trial in northern Red 
Sea. All of the synthesised paints presented significant antifouling properties after 5 months of exposure in real sea water conditions and the dual antifouling effect of the nanoparticles was confirmed.

\section{Introduction}

The definition of biofouling or biological fouling is the accumulation of microorganisms, plants, algae or animals on wetted surfaces. From the first minute of immersion of a clean surface in natural seawater, it immediately starts to adsorb a molecular conditioning film consisted of dissolved organic materials ${ }^{1}$. After that initial stage, the surface colonisation by a wide range of organisms depends on their relative rate of attachment and surface exploitation .,2 According to the literature, surface colonisation follows a linear "successional model" in which biofilm formation starts within a week by spores of macroalgae (seaweeds), fungi and protozoa and after that within several weeks by larvae of invertebrates such as barnacles.$^{3,4}$ This classical view is a simplification as motile spores of seaweeds are capable of settling within minutes on a clean surface and larvae of some species of barnacles or bryozoans settle within a few hours of immersion. ${ }^{1}$ The distinction between the different fouling stages comprises "microfouling", "soft macrofouling" and "hard macrofouling". Microfouling usually referred to microorganisms such as protozoa, bacteria and diatoms, forming complex biofilms during the first $24 \mathrm{~h}$ of immersion. Soft macrofouling comprising the formation of microscopically visible algae, seaweeds and invertebrates such as soft sponges, corals, tunicates and anemones after 2 or 3 weeks of immersion. Hard macrofouling refers to the colonisation of mussels, barnacles, ascidians, etc.

Biofouling has serious effects in various sectors of maritime industry. It is the main expense of maintenance for any immersed man-made surface such as buoys, membrane bioreactors and 
desalination units, power plants' cooling water systems and oil pipelines. Furthermore, it has significant effects on the aquaculture industry especially in marine finfish aquaculture where the use of sea cages is necessary. In the ship industry, the adhesion of marine algae and barnacles on the hulls of the ships makes them rough and causes increased erosion, reduction of speed, increased fuel consumption (up to $40 \%$ ) and, consequently, increases the air pollution and $\mathrm{CO}_{2}$ emission. ${ }^{5,6}$ As the diversity of fouling organisms is vast and the range of adhesion mechanisms correspondingly broad, it is a major challenge the creation of an effective fouling resistant coating.

The majority of the coatings in the past were biocide-based self-polishing coatings and their main component was the tributyltin (TBT), an efficient and versatile biocide. ${ }^{7}$ The main applications of this biocide were for wood preservation, antifungal applications in industrial processes, marine antifoulings and as pesticides. However, due to their toxicity to humans and their negative impact on the environment, TBT compounds became prohibited worldwide after 1 January 2003 and their use as ship coating became completely banned after 1 January 2008 by the International Convention on the Control of Harmful Anti-fouling Systems on Ships of the International Maritime Organization. ${ }^{89}$ Subsequently, antifouling manufacturers have been forced to urgently develop new and more environmentally friendly antifouling paints. Almost all the antifouling paints today contain copper as their primary active ingredient which is well known for more than 2000 years from ancient years. Since 300 B.C., Greeks and Romans used copper in the bronze-shod rams of their warships and copper nails to secure the lead sheathing. ${ }^{7,10}$

To date, the working principle of the most of paint systems applied is based on slow release of biocide compounds. This operating principle has drawbacks for the paint systems ${ }^{11}$. Usually, the 
main active ingredient dissolves away and the paint loses its effectiveness but the binder stays behind. $^{7}$ Furthermore, as more coats are applied, the dead binder layers get thicker and thicker until they start to flake off in large chunks. Thus, it is very difficult and expensive to remove the old paint, and as a consequence, this type of coatings has been put aside. In addition, the antifouling release materials like perfluorinated or silane containing compounds were directly introduced into the coating thereby influencing other coating properties like corrosion protection. One approach to eliminate some of these drawbacks is the encapsulation, a methodology that has been applied into effective delivery systems for various applications in biotechnology, energy storage systems, protection against corrosion, pharmaceutics and food industry. ${ }^{12-14}$ Commonly used technologies to encapsulate small molecules such as biocides include the Layer-by-Layer method and physical adsorption into porous materials. ${ }^{15-17}$ By using proper carriers for encapsulation, it is possible to decrease and control the release rate of biocides and provide an effective protection from the surrounding environment. ${ }^{18,19}$

The quaternary ammonium salts (QASs) have been indexed as antimicrobial compounds for more than 70 years. $^{20,21}$ They have been used against the growth of a broad range of microorganisms in several applications including food and pharmaceutical products, antiseptics, disinfectants, biocides, fungicides, cosmetics and water treatment. ${ }^{22,23}$ The QASs are positively charged cationic compounds and the bacterial cell membranes are negatively charged. As a consequence of electrostatic interactions, the QASs can be adsorbed on the cell surfaces and diffuse through the cell wall. The next step is their binding to the cytoplasmic membrane causing its disruption and release of $\mathrm{K}^{+}$ions and constituents of the cytoplasmic membrane which leads to cell death. ${ }^{24,25}$ The effectiveness of the QASs in killing a wide range of microorganisms as antimicrobial agents attracted the interest of scientists. In several studies, QAS were used to 
functionalise surfaces of different materials as antimicrobial treatment. ${ }^{24-28}$ The advantage of the QAS-modified materials in comparison with the classic biocide-release coatings can be attributed to their attachable nature to the fillers of the coatings which allows a permanent antifouling effect of the coating without the release of the biocide material. There are several parameters which influence the interactions between the quaternary ammonium moieties and the cell membranes and therefore their biocidal activity. The effectiveness of a specific QAS is microorganism dependent since there are variations to the composition and structure from one microorganism to another ${ }^{29}$. An important parameter for the efficiency of the QAS is the alkyl chain length of their general molecular formula $\left(\mathrm{NR}_{4}{ }^{+}\right)$. It has been found that QAS with alkyl chain length from $\mathrm{C} 8$ and higher present high antimicrobial activity. ${ }^{30}$

This work is focused on the development of functional fillers for antibacterial/antifouling coatings with advanced performance compared to the current state of the art coatings. Usually in the literature describing biomedical and antifouling systems, non-porous silica nanoparticles are used only for surface modifications and mesoporous silica nanoparticles (MSNs) only for encapsulation. However, to the best of our knowledge, this is the first study that describes the development of dual-function antibacterial/antifouling MSNs based on the combination of two strategies (surface modification and encapsulation) in one system. Through our approach, we are aiming to demonstrate for first time the idea of an easy, simple and straightforward method for the development of dual-functional nanoparticles with long-lasting effect by using established and effective antibacterial/antifouling compounds. For this purpose, spherical mesoporous MCM-48 silica nanoparticles with a dual synergetic antimicrobial effect were synthesised and tested against Gram positive and Gram negative bacteria. For the preparation of MSNs, the modified Stöber's method was used according to Schumacher et al. because it is an easy and fast 
way to obtain MCM-48 at room temperature. ${ }^{31,32}$ MCM-48 spherical nanoparticles were chosen for two main reasons. Firstly, their interwoven, branched 3D mesostructure makes them excellent candidates for the loading and release of biocides. Secondly, this is the first study that reports on the surface modification of MCM-48 with QAS.

The surface of the nanoparticles was modified with two different QASs, dimethyloctadecyl [3(trimethoxysilyl) propyl] ammonium chloride and dimethyltetradecyl [3-(triethoxysilyl) propyl] ammonium chloride. This modification provides the coatings the necessary hydrophobicity and antifouling properties, biocidal activity against marine microorganisms and resistance to microfouling in seawater immersion. Afterwards, the QAS-modified MSNs were loaded with a liquid biocide (Parmetol S15) for dual antifouling effect. The active ingredient for the Parmetol S15 is the 4, 5-dichloro-2-octyl-4-isothiazolin-3-one (DCOIT), a highly hydrophobic compound with good biodegradability and low water solubility. The DCOIT received the first Environmental Protection Agency (EPA) Green Chemistry award in 1996 for replacing the TBT in antifouling products and has been successfully used in encapsulation techniques for microcapsules with antifouling properties. ${ }^{33}$ The final synthesised materials with the encapsulated biocide exhibit superior performance in the antibacterial tests due to the dual antimicrobial effect from the modified surface with QAS and from the encapsulated biocide in the mesostructure of the nanoparticles (NPs). Through our approach, we are aiming to prevent the early stages of the biofilm and biofouling formation where everything starts with the attachment of microorganisms on surfaces. Modified nanoparticles with high antibacterial performance are great potential fillers for biofilm and fouling resistant coatings. The modified MSNs can provide permanent antibacterial properties to the coating formulations even after the release of the biocide due to the existence of the chemically attached QAS groups on the NPs 
surface. A five months field test trial was also conducted in order to examine the antifouling performance of the synthesised modified nanoparticles after addition into coating formulations. PVC panels were coated with the synthesised paints and after five months of exposure in real seawater conditions the biofouling coverage was significantly lower compared to the panels coated with the biocide-free pristine paint.

\section{Results and discussion}

Synthesis and characterisation of MCM-48. MCM-48 spherical MSNs were chosen as carriers for the antifouling/antibacterial compounds. MCM-48 can be easily functionalised as they exhibit high surface reactivity due to the existence of the hydroxyl groups on their surface. In addition, their interwoven, branched 3D mesoporous structure makes them excellent candidates for the loading and release of biocides. For the synthesis of the MSNs was used the modified Stöber's method as reported in Schumacher et al. ${ }^{31,32}$ The powder XRD spectrum of the synthesised MCM-48 after calcination is similar to the literature data (Figure 1) ${ }^{31}$ The samples exhibit five Bragg diffraction peaks in the range $2 \theta=1.5-10^{\circ}$ which correspond to the planes (211), (220), (421), (332) and (431). The diffraction peaks can be assigned to the typical cubic space group Ia3d characteristic for mesoporous materials with 3D cubic structure. The planes (211) and (220) are intense and sharp which is an indication of high ordering of our synthesised MCM-48.

The low-temperature nitrogen isotherms can provide accurate and reliable information associated with the specific surface area and the quality of the mesoporous structure of solid materials. ${ }^{34}$ The sample presented type IV isotherm according to the Brunauer-Deming-DemingTeller classification which is characteristic for MCM-41 and MCM-48 mesoporous materials. ${ }^{35}$ 
From the adsorption and desorption isotherm no hysteresis was observed and they showed a sharp capillary condensation step which provides information for uniform pore channels and narrow pore size distribution (Figure S1a). ${ }^{36-39}$ The specific surface area was calculated according to the Brunauer - Emmett - Teller (BET) method and the mesoporous MCM-48 samples exhibited high values of $\mathrm{S}_{\mathrm{BET}}$ in a range from 1180 to $1300 \mathrm{~m}^{2} / \mathrm{g}{ }^{40}$ The pore size distribution and the total pore volume of the synthesised MCM-48 materials were calculated from the nitrogen adsorption isotherms by using the nonlocal density functional theory (NLDFT) method. ${ }^{41-43}$ It has been well documented that the NLDFT model is more accurate and rigorous approach for the interpretation of the nitrogen isotherms and the calculation of mesoporous structure properties for MCM-48 materials..$^{31,32,44,45}$ The synthesised MCM-48 material presented high total pore volume of $0.8 \mathrm{~cm}^{3} / \mathrm{g}$ at 0.99 relative pressure. Furthermore, the material under study exhibited narrow pore size distribution as expected due to the sharp capillary condensation step (Figure S1b). The average pore diameter is $3.2 \mathrm{~nm}$ as calculated from the NLDFT method.

Scanning electron microscopy (SEM) was used to observe the morphology of the samples. The SEM images of the mesoporous MCM-48 after calcination at $550{ }^{\circ} \mathrm{C}$ are shown in Figure 2. The samples showed spherical morphology in the range of $150-600 \mathrm{~nm}$. In the reaction mixture, the presence of ammonia works as a morphological catalyst responsible for the spherical size of the particles. ${ }^{46}$ As can been seen from the SEM images, some of the particles are aggregated and fused together but most of them are single monodispersed particles.

Surface modification with QAS. MCM-48 modified with QASs were characterised by TGA, FTIR, elemental analysis and $\zeta$-potential measurements. The FTIR spectra for the pristine MCM48, QC14 and QC18 modified MCM-48 are shown in Figure 3. All samples present the characteristic absorption peaks for the $\mathrm{SiO}_{2}$ materials: Si-O-Si bending vibration $\left(465 \mathrm{~cm}^{-1}\right)$, Si- 
O-Si symmetric stretching $\left(800 \mathrm{~cm}^{-1}\right)$, Si-OH asymmetric vibration $\left(940 \mathrm{~cm}^{-1}\right)$, Si-O asymmetric vibration $\left(1080 \mathrm{~cm}^{-1}\right)$, H-O-H bending vibration $\left(1635 \mathrm{~cm}^{-1}\right)$ and $-\mathrm{OH}$ stretching $\left(3400 \mathrm{~cm}^{-1}\right)$. However, the QAS-modified samples showed three additional peaks. These peaks represent the alkyl chain of the QASs and prove that the silica nanoparticle was successfully decorated by QAS. The peak at $1465 \mathrm{~cm}^{-1}$ corresponds to the $\mathrm{C}-\mathrm{H}$ bending vibration and the peaks at 2850 and $2950 \mathrm{~cm}^{-1}$ correspond to the $\mathrm{C}-\mathrm{H}$ stretching. Furthermore, the broad $-\mathrm{OH}$ peak at $3400 \mathrm{~cm}^{-1}$ for the pristine MCM-48 is more intense compared to the QAS-modified MCM-48 because of the free $-\mathrm{OH}$ groups on the surface and within the pore channel system of pristine MCM- 48 . There is also a significant reduction in the intensity of the O-H peak in the QAS-modified MCM-48 spectra (Supporting Information, Figure S2). Therefore, the H-O-H bending vibration (1635 $\mathrm{cm}^{-}$ ${ }^{1}$ ) and $-\mathrm{OH}$ stretching $\left(3400 \mathrm{~cm}^{-1}\right)$ for the QAS-modified MCM-48 will give very low signal as most of the available hydroxyl groups reacted with the QAS.

Further information about the surface properties of the modified and unmodified materials was obtained via $\zeta$-potential measurements and elemental analysis (Table 1). The surface of the pristine MSN is positively charged at $\mathrm{pH}$ below its isoelectric point (IEP $=\mathrm{pH} 2.0)$ and negatively charged above the IEP. ${ }^{47}$. At the $\mathrm{pH}$ range from 1.5 to 9.5 , the $\zeta$-potential for the QAS modified nanoparticles was shifted significantly to positive values due to the presence of the positively charged quaternary ammonium groups on the surface of the materials instead of the negatively charged hydroxyl groups. Taking into consideration the high values of $\zeta$ - potential for the QAS modified materials (between $+40 \mathrm{mV}$ and $+60 \mathrm{mV}$ ), we can hypothesise that the modified particles have good stability in colloidal suspensions. Further confirmation of the successful surface modification comes from the elemental analysis. The QC18 and QC14 modified particles showed significant increment of $\% \mathrm{C}, \% \mathrm{H}$ and $\% \mathrm{~N}$ compared to the pristine MCM-48 due to 
the alkyl functional groups and the positively charged nitrogen groups of the covalently attached QAS moieties (Table 1).

The thermogravimetric analysis (TGA) was used to evaluate the amount of QAS grafted on the surface of the MSNs when heated under $\mathrm{N}_{2}$ atmosphere up to $800{ }^{\circ} \mathrm{C}$ (Figure 4). The pristine MCM-48 was calcinated up to $800{ }^{\circ} \mathrm{C}$ and its weight decreased only by $4 \%$ mainly because of the decomposition of silanol groups and the existence of some humidity inside the material. The silica NPs have very high thermal stability and almost keep their weight constant up to $800{ }^{\circ} \mathrm{C}$ at least. However, when QC18 or QC14 modified MCM-48 nanoparticles were calcinated to 800 ${ }^{\circ} \mathrm{C}$, they lost $28.3 \%$ and $19.2 \%$ of their initial weight respectively. Both QC18- and QC14modified MCM-48 presented 3\% weight loss between $30{ }^{\circ} \mathrm{C}$ and $130{ }^{\circ} \mathrm{C}$ because of humidity and decomposition of unreacted silanol groups. The further $25.3 \%$ or $16.2 \%$ weight loss between 200 ${ }^{\circ} \mathrm{C}$ and $800{ }^{\circ} \mathrm{C}$ is attributed to the calcination of the attached QC18 or QC14 groups respectively.

Significant differences between the two types of modified materials at the TGA and the $\zeta$ potential measurements and the elemental analysis are caused by two main reasons. Firstly, there are four extra $-\mathrm{CH}_{2}$ groups in the alkyl chain of the QC18 molecule compared to the QC14 molecule. Therefore, we expected higher weight loss and higher \% of $\mathrm{C}$ and $\mathrm{H}$ for the QC18modified material at the TGA measurements and elemental analysis. Secondly, the QC18 compound was commercially available at $99 \%$ purity while the QC14 compound is commercially unavailable and as a result was synthesised in-house. The dimethyltetradecyl [3(triethoxysilyl) propyl] ammonium chloride (QC14) was synthesised by reacting $1.97 \times 10^{-2}$ moles of $\mathrm{N}$, N-dimethyltetradecylamine with $2.08 \times 10^{-2}$ moles of 3-chloropropyltriethoxy silane at $110{ }^{\circ} \mathrm{C}$ for $48 \mathrm{~h}$. After the reaction, the colour of the solution became yellow-brown and the viscosity increased confirming the quartenisation reaction (Supporting Information, Figure S3 
and S4). ${ }^{1} \mathrm{H}-\mathrm{NMR}$ spectroscopy was used in order to confirm the successful synthesis of the QC14 (Supporting Information, Figure S5). The ${ }^{1} \mathrm{H}-\mathrm{NMR}$ spectrum of the product (QC14) showed two additional peaks at $3.38 \mathrm{ppm}\left[\left(-\mathrm{N}^{+}-\left(\mathrm{CH}_{3}\right)_{2}\right]\right.$ and $3.46 \mathrm{ppm}\left(-\mathrm{N}^{+}-\mathrm{CH}_{2}\right)$ because of the quaternization. However, the presence of the peaks at $3.54 \mathrm{ppm}\left(-\mathrm{CH}_{2}-\mathrm{Cl}\right)$ and $2.2 \mathrm{ppm}[-\mathrm{N}-$ $\left.\left(\mathrm{CH}_{3}\right)_{2}\right]$ after 48 hours of reaction indicate that there are residual N,N-dimethyltetradecylamine and 3-chloropropyltriethoxy silane. Thus, during the surface modification of the MCM-48 with QC14, the available silanol groups on the MCM-48 surface can react both with the 3chloropropyltriethoxy silane and the QC14. As a result, the surface of the QC14-modified MCM48 is covered with QC14 molecules and 3-chloropropyltriethoxy silane molecules. Our hypothesis is confirmed by the decreased amount of nitrogen in elemental analysis, the decreased positive values of $\zeta$-potential measurements and the lower weight loss on the TGA curve for the QC14 functionalised silica compared to the QC18 functionalized silica.

Encapsulation of biocide. Encapsulation is an important technology that finds applications in pharmaceutical industry, food industry, energy storage systems, etc. by using materials such as mesoporous silica nanoparticles, polymeric capsules, hydrogels, etc. as hosts. ${ }^{48,49}$ In our approach for the encapsulation of Parmetol S15 biocide, the two different types of modified MCM-48 were dispersed directly via sonication in the commercially available liquid biocide Parmetol S15 and left under vacuum overnight. After the recovery of the solid materials with the encapsulated biocide, the samples were tested with the TGA and the amount of the loaded biocide was measured. The TGA curves of the QAS-modified MCM-48 loaded with the Parmetol S15 were compared with the TGA curves of the unloaded QAS-modified MCM-48 (Figure 5). The loaded QC18-modified MCM-48 with Parmetol showed 26.9\% wt. additional weight loss in comparison with the unloaded QC18-modified MCM-48 due to the encapsulated 
biocide. The loaded QC14-modified MCM-48 with Parmetol exhibited 31.5\% wt. further weight loss in comparison with the unloaded QC14-modified MCM-48, which is also attributed to encapsulated biocide. The TGA curves confirmed the successful encapsulation of the biocide inside the MSNs. During the surface modification, the $-\mathrm{Si}\left(\mathrm{OCH}_{3}\right)_{3}$ groups of the quaternary silane are covalently bonded with $-\mathrm{OH}$ groups close to the opening of the pores thus blocking some of the pores for biocide ingress. Moreover, in the case of the QC18 modified MSNs, the blockage of the pores is higher due to its larger molecular size. This can explain the $4.6 \% \mathrm{wt}$. lower amount of encapsulated biocide inside the QC18-modified MCM-48 compared to the amount encapsulated inside the QC14-modified MCM-48.

Antibacterial performance. For the evaluation of the antibacterial activity of synthesised materials, $22 \mathrm{~mm} \times 22 \mathrm{~mm}$ glass slides were coated with a spin coater by using $1 \mathrm{~mL}$ of these particles dispersed in ethanol solution (2\% wt) and tested against Gram-negative E. Coli and Gram-positive $S$. aureus. The pristine MCM-48 was used as a negative control since no antibacterial activity is expected from this material. The relative number of viable bacteria adhered on the glass slide coated with pristine MCM-48 was defined as $100 \%$. As can been seen from the Figure 6, the QC18 and QC14 modified MSNs reduced the number of the viable bacteria $E$. Coli by $89 \%$ and $77 \%$ respectively compared to the pristine MCM- 48 while the two types of modified MSNs with the encapsulated Parmetol S15 presented excellent and enhanced antibacterial performance by killing all the exposed bacteria during the test resulting in $100 \%$ reduction compared to the pristine MCM-48.

Figure 7 shows the antibacterial performance of the pristine and modified MCM-48 against the gram positive bacteria $S$. aureus. The QC18 and QC14 modified MCM-48 reduced the relative number of viable bacteria by $94 \%$ and $78 \%$ respectively. The QC18 and QC14 modified 
MCM-48 and loaded with the additional biocide Parmetol S15 exhibited 100\% reduction by killing all the exposed bacteria at the end of the experiment. In the antibacterial tests against both types of bacteria, there is a statistically significant $(p<0.0005)$ increase in antibacterial activity when the pristine MCM-48 are either modified only with QAS or modified with QAS and loaded with Parmetol S15.

It is noteworthy that the QC18 modified MSNs presented higher antibacterial performance against E. coli and S. aureus in comparison with the QC14 modified MSNs. As mentioned previously, the surface of the QC14-modified MCM-48 is covered with QC14 molecules and 3chloropropyltriethoxy silane molecules because after the quaternisation reaction of the QC14 there was residual 3-chloropropyltriethoxy silane. Therefore, the QC18 modified MCM-48 have higher amount of quaternary ammonium groups than the QC14 modified MCM-48 which is confirmed by the elemental analysis (Table 1). There are several studies supporting that increased numbers of quaternary ammonium groups results in increasing the antibacterial performance of the compound. ${ }^{50,51}$ In addition, according to the killing mechanism of the QAS, the longer the alkyl chain length of the quaternary compound the easier it penetrates the cell membrane and causes the death of the bacteria. As a consequence, the QC18 modified MCM-48 exhibited increased antibacterial performance compared to the QC14 modified MCM-48. The results from the antibacterial tests against $S$. aureus and $E$. coli come as a confirmation of the dual synergetic antibacterial effect of the QAS-modified MSNs with the encapsulated Parmetol S15. The antibacterial effect will be permanent even after the release of the encapsulated biocide because of the presence of the covalently attached QAS on the nanoparticle surface.

Antifouling performance of coated PVC panels with the synthesised paints: A panel field test took place in northern Red Sea, Eilat, Israel for five months period in order to evaluate the 
antifouling efficacy of the modified MCM-48 nanoparticles incorporated in coatings. PVC panels coated with Pristine Paint (biocide-free paint) were used as control samples. QC18modified MCM-48 and loaded with Parmetol S15, QC14-modified MCM-48 and loaded with Parmetol S15, QC18-modified MCM-48 and QC14-modified MCM-48 were added in 5\% wt. concentration into the Pristine Paint and the Paints 1-4 were synthesised respectively. Figure 8 illustrates photographs of the exposed coated panels in the first day of deployment and after five months of exposure. At the end of the field test, the control sample coated with the Pristine Paint presented 39\% biofouling coverage while the Paints 1-4 presented significantly lower biofouling coverage below $10 \%$. In particular, Paints 1 and 2 containing the modified nanoparticles with the dual effect illustrated superior performance with $6.7 \%$ and $7.8 \%$ biofouling coverage due to the encapsulated biocide into the QAS modified nanoparticles confirming their enhanced antifouling effect. Furthermore, Paint 3 and 4 containing the QAS modified nanoparticles exhibited 8.4\% and $9.8 \%$ biofouling coverage respectively suggesting that even after the release of the encapsulated biocide, the QAS groups on the surface of the nanoparticles will continue to provide antifouling properties to the coating formulations.

\section{Conclusions}

The MCM-48 mesoporous silica nanoparticles with average size $400 \mathrm{~nm}$ were successfully synthesised as showed by the XRD, SEM and BET measurements. The synthesised materials presented XRD patterns characteristic for highly ordered 3D mesoporous structures. On the second step, the mesoporous silica nanoparticles surface modified with quaternary ammonium salts (either dimethyloctadecyl [3-(trimethoxysilyl) propyl] ammonium chloride (QC18) or dimethyltetradecyl [3-(triethoxysilyl) propyl] ammonium chloride (QC14)) were successfully 
synthesized revealing strong covalent bonds between the quaternary ammonium salts and the surface of the nanoparticles. The surface functionalisation was confirmed by FTIR, TGA, elemental analysis and $\zeta$-potential measurements. The FTIR spectra for the modified materials showed 3 additional peaks confirming the presence of $\mathrm{C}-\mathrm{H}$ bonds due to the attached QASs on the MCM-48 surface. The TGA curves showed 19\%-28\% wt. further weight loss for the modified MSNs due to the QASs calcination. The $\zeta$-potential presented significant change of the surface charges from negative to positive $(>80 \mathrm{mV})$ after the modification of the negatively charged silica with positively charged quaternary ammonium groups. To achieve dual synergetic effect, the synthesised materials were loaded with one additional biocide for an enhanced dual antibacterial/antifouling effect.

The encapsulation of the biocide Parmetol S15 was confirmed by the TGA curves where the loaded materials presented 31.5\% wt. (QC18-modified) and 26.9\% wt. (QC14-modified) of weight loss compared to the unloaded materials. In the antibacterial tests, all of the synthesised modified nanoparticles exhibited high antibacterial performance confirming their dual activity. In the case of the QAS-modified MCM-48 loaded with the biocide Parmetol S15, all the exposed bacteria were dead at the end of the experiments for both antibacterial tests against Gram negative and Gram positive bacteria. Furthermore, the QAS-modified MCM-48 presented 77$89 \%$ reduction against gram negative bacteria and $78-94 \%$ reduction against gram positive bacteria. Finally, the modified nanoparticles were incorporated into coating formulations and their antifouling activity was assessed in a field test trial. All of the synthesised paints illustrated significant antifouling activity and the biofouling coverage was considerably lower compared to the biocide-free Pristine Paint. The results from the field test provide a confirmation of the dual 
antifouling activity of the synthesised nanoparticles that can be used as potential functional fillers for tin-free alternative paints in marine industry.

Through our approach, we propose for first time a quick and effective way to synthesise antibacterial nanoparticles with dual synergetic effect and excellent and permanent performance. In our studies, we used a commercially available biocide for the encapsulation. However, due to the hydrophobic nature of the QAS-modified MSNs, other biocides or antibacterial agents can be used for the encapsulation. The modified particles with the dual synergetic effect can be excellent carriers of antibacterial/antifouling compounds for coating formulations in several applications such as hospitals, marine industry, etc.

\section{Materials and methods}

Materials: For the synthesis of the MSNs, tetraethyl orthosilicate (TEOS 98\%, SigmaAldrich) was used as the source of silica and hexadecyltrimethylammonium bromide (CTAB 99\%, Sigma-Aldrich) was used as surfactant (structure directing agent). Ethanol (99.8\%, SigmaAldrich) and ammonium hydroxide (32\%, Merck) were used to carry out the synthesis of MSNs. Dimethyloctadecyl [3-(trimethoxysilyl) propyl] ammonium chloride (QC18 60\% in methanol, Acros Organics) and dimethyltetradecyl [3-(triethoxysilyl) propyl] ammonium chloride (QC14) were the quaternary ammonium salts used for the surface modification of the MSNs. The biocide for the loading of the MSNs was Parmetol S15 (Schulke). The chemical composition of Parmetol is similar to SeaNine, as in both biocides the active compound is the 4,5-dichloro-2-octyl-4isothiazolin-3-one (DCOIT). Parmetol S15 can be easier encapsulated into the mesostructure of our MSMs due to its better compatibility with the hydrophobic pore channel system of the QASmodified MSNs. 
Synthesis of MSNs with average size of $400 \mathrm{~nm}$ : The modified Stöber's method was used for the synthesis of MSNs as reported in Schumacher et al.. ${ }^{31,32}$ According to this method, spherical MCM-48 can be synthesized at room temperature. In a typical synthesis route, hexadecyltrimethylammonium bromide $(2.6 \mathrm{~g}, 6.6 \mathrm{mmoles})$ was dissolved in a mixture of deionized water $(120 \mathrm{~mL})$ and pure ethanol $(50 \mathrm{~mL}, 0.87$ moles $)$ and ammonium hydroxide (12 $\mathrm{mL}$ of $32 \%$ wt. solution) was added to the surfactant solution. The solution was stirred for 10 minutes at $600 \mathrm{rpm}$ and tetraethyl orthosilicate $(3.4 \mathrm{~g}, 16$ mmoles) was added at once during stirring. After stirring for 16 hours at room temperature the resulting solid was recovered by filtration, washed twice with ethanol, twice with distilled water and dried in air at ambient conditions. The organic template was removed by calcination in a muffle furnace at $550{ }^{\circ} \mathrm{C}$ for 6 $\mathrm{h}$ after heating with a ramp of $1{ }^{\circ} \mathrm{C} / \mathrm{min}$.

\section{Synthesis of dimethyltetradecyl [3-(trimethoxysilyl) propyl] ammonium chloride (QC14):}

The dimethyltetradecyl [3-(triethoxysilyl) propyl] ammonium chloride (QC14) was synthesised by reacting $4.76 \mathrm{~g} \quad \mathrm{~N}, \mathrm{~N}$-dimethyltetradecylamine $\left(\begin{array}{lllllll}1.97 & \mathrm{x} & 10^{-2} & \text { moles}\end{array}\right)$ with $5 \mathrm{~g}$ 3chloropropyltriethoxy silane $\left(2.08 \times 10^{-2}\right.$ moles $)$ in a $20 \mathrm{~mL}$ vial. The vial containing the reaction mixture was purged with nitrogen for $10 \mathrm{~min}$ before sealing. The quartenisation reaction was carried out at $110{ }^{\circ} \mathrm{C}$ for $48 \mathrm{~h}$ using magnetic stirring. After $48 \mathrm{~h}$, the reaction mixture was cooled at room temperature before adding $9.76 \mathrm{~g}$ of methanol to produce $50 \mathrm{wt} \%$ solution of dimethyltetradecyl [3-(trimethoxysilyl) propyl] ammonium chloride (QC14) in methanol. ${ }^{52}$

Surface modification of the MSNs: The QAS-modified MCM-48 was prepared by hydrolysis and condensation reactions between the quaternary ammonium salt and the hydroxyl groups on the surface of the MCM-48 (Supporting Information, Figure S2). For the surface modification, 1 $\mathrm{g}$ of calcinated MCM-48 powder was dispersed in a mixture of ethanol $(50 \mathrm{~mL})$ and deionised 
water $(50 \mathrm{~mL})$ using sonication bath. To the homogeneous solution, $0.5 \mathrm{~mL}$ of either QC18 or QC14 was added and stirred for 24 hours at room temperature. The modified NPs were collected with centrifugation, washed twice with ethanol and twice with water to remove the residual reagents and dried to ambient conditions.

Loading of the modified MSNs with biocide: Before loading the modified MSNs, the samples were dried at $100{ }^{\circ} \mathrm{C}$ under vacuum in order to remove water and air from the mesoporous structure. Subsequently, modified MSNs $(0.20 \mathrm{~g})$ were dispersed directly in the biocide Parmetol S15 $(20 \mathrm{~mL})$ and stirred under vacuum overnight. The solid was recovered by filtration and gently washed on the filter paper dropwise with ethanol. The loaded NPs were dried at ambient conditions.

Characterisation methods: The powder XRD patterns were obtained on a Bruker diffractometer using $\mathrm{CuKa}$ radiation. The morphology of the samples was observed using scanning electron microscope JEOL 7001F. The thermo gravimetric analysis (TGA) curves were obtained at Linseis STA PT-1000 instrument with calcination temperature up to $800{ }^{\circ} \mathrm{C}$ under nitrogen. The chemical structure of the modified NPs was confirmed by recording their FTIR spectra in the wavenumber range from 4000 to $400 \mathrm{~cm}^{-1}$ using the instrument Bruker Tensor II. The $\zeta$-potential for pristine and modified samples (dispersed in aqueous solutions at ambient conditions) was measured using Malvern Zetasizer Nano ZS. Nitrogen adsorption isotherms were measured at $77 \mathrm{~K}$ on a Quadrasorb SI analyser (Quantachrome Instruments). Before the measurements, the samples were outgassed for 20 hours at $423 \mathrm{~K}$. The elemental analysis values were obtained using Flash EA 1112 organic elemental analyser.

Antibacterial activity of pristine and modified MCM-48: S. aureus (ATCC 25923) and E. coli (ATCC 10536) cultures were grown overnight at $37{ }^{\circ} \mathrm{C}$ in sterilised nutrient broth medium 
(NB). The bacterial cells were centrifuged and the NB was replaced by a sterile 500 fold diluted NB (1/500 NB). For the tests against Gram-negative E. coli and Gram-positive S. aureus, $22 \mathrm{~mm}$ x $22 \mathrm{~mm}$ glass slides were coated by $1 \mathrm{~mL}$ of either pristine or modified nanoparticles dispersed in ethanol solution ( $2 \% \mathrm{wt}$.) with a spin coater. The samples used for the antibacterial tests were: pristine glass, pristine MCM-48, QC18-modified MCM-48 and loaded with Parmetol S15, QC14-modified MCM-48 and loaded with Parmetol S15, QC18-modified MCM-48 and QC14modified MCM-48. The pristine MCM-48 was used as a negative control since no antibacterial activity is expected from this material. The spin-coated glass slides were UV sterilised for 1 hour and were inoculated with $50 \mu \mathrm{L}$ of $10^{5}$ colony forming unit per $\mathrm{mL}(\mathrm{CFU} / \mathrm{mL})$ of either $S$. Aureus or E. Coli solution. Each sample was covered with a $20 \mathrm{~mm}$ x $20 \mathrm{~mm}$ sterile glass cover slip and was incubated at $37^{\circ} \mathrm{C}$ for 3 hours. Then, each sample was introduced in a $50 \mathrm{~mL}$ falcon tube containing $10 \mathrm{~mL}$ of phosphate-buffered saline solution (PBS) and was vortexed prior to spreading $100 \mu \mathrm{L}$ of the bacterial solution on an agar plate. Inoculated agar plates were incubated overnight and the CFU/mL was counted. Each sample type was studied in triplicate following this method. One-way analysis of variance (ANOVA) was used to compare mean values in order to determine the equivalence of variance between the samples. Significance between the samples was determined using the Bonferroni multiple comparison test where a value of $p<0.05$ was taken as statistically significant.

\section{Antifouling activity of coating formulations containing modified MCM-48 (Field Test):}

For the preparation of the test paints, as-synthesised modified MCM-48 were suspended mechanically via a homogeniser in a polymeric paint matrix. The polymeric paint matrix (Pristine Paint) was developed specifically for the EU FP7 BYEFOULING project by Jotun and it is a solvent based biocide-free coating formulation. The concentration of the nanoparticles in 
the paints was adjusted to $5 \% \mathrm{wt}$ and four different paint formulations were synthesised. Paint 1 : Pristine Paint $+5 \%$ wt. QC18-modified MCM-48 and loaded with Parmetol S15, Paint 2: Pristine Paint $+5 \%$ wt. QC14-modified MCM-48 and loaded with Parmetol S15, Paint 3: Pristine Paint $+5 \%$ wt. QC18-modified MCM-48 and Paint 4: Pristine Paint + 5\% wt. QC14modified MCM-48.

PVC panels of 7 x $10 \mathrm{~cm}$ coated with five different types of paints (Pristine Paint and Paints 14) were immersed in the Red Sea, Eilat, Israel for a five months field test trial for a realistic assessment of their antifouling properties. The coated PVC panels were placed on a floating structure made of a stainless steel frame on which two PVC boards were attached and submerged at a depth of 8-9 m (Figure S7). Each panel was attached by nuts to two stainless steel bolts. The panels were underwater photographed during exposure period and the percentage of fouling covering the panels was determined by using the image analysis software Image $J .^{53}$ The quantification of the fouling and the biofilm provided a round overview of the coatings performance. 


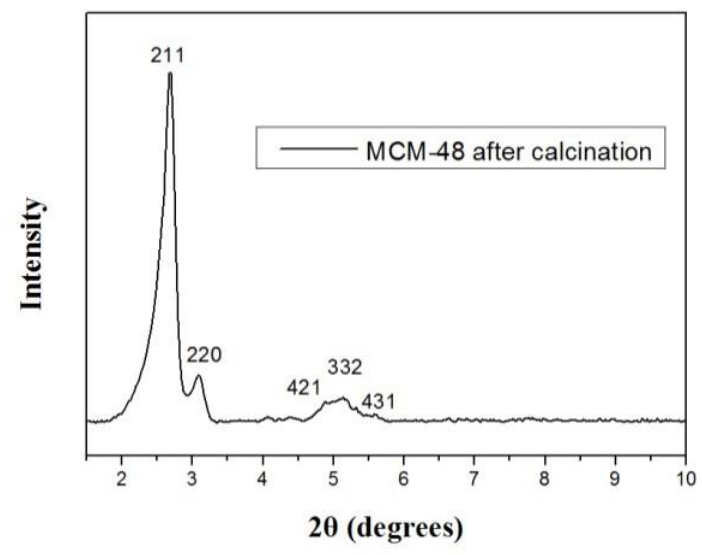

Figure 1. XRD patterns for the synthesised MCM-48 mesoporous nanoparticles, planes (211), (220), (421), (332) and (431) correspond to the cubic space group Ia3d.
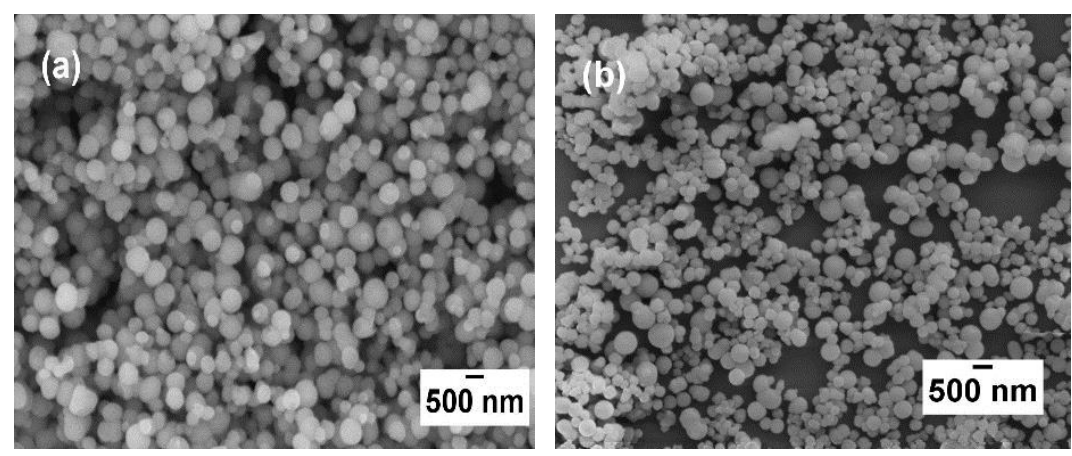

Figure 2. SEM images for the synthesised (a) pristine MCM-48 and (b) QAS-modified MCM48. 


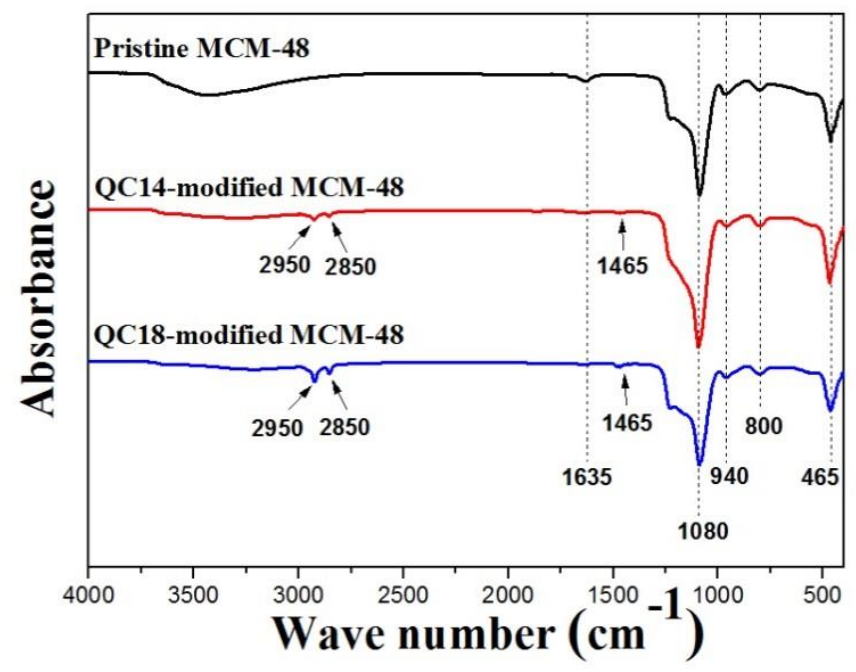

Figure 3. FTIR spectra for pristine MCM-48, QC14 and QC18 modified MCM-48.

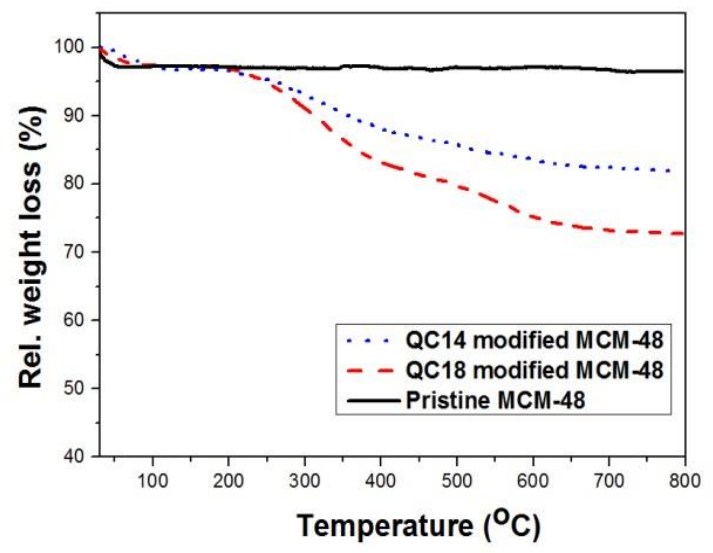

Figure 4. TGA curves for the pristine MCM-48, QC18-modified MCM-48 and QC14-modified MCM-48. 
(a)

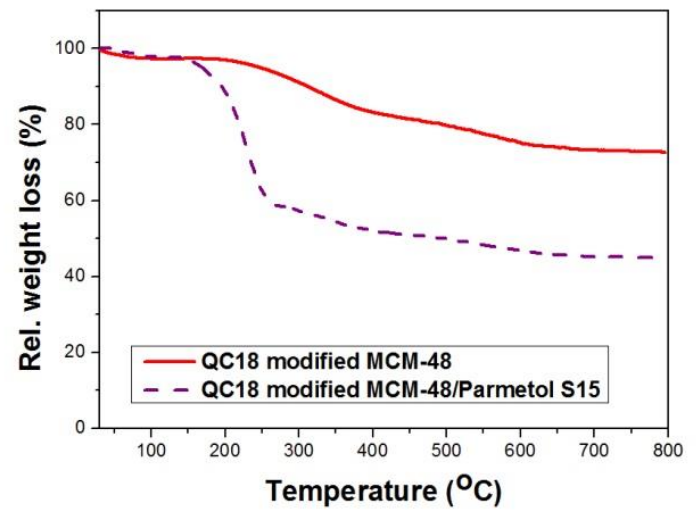

(b)

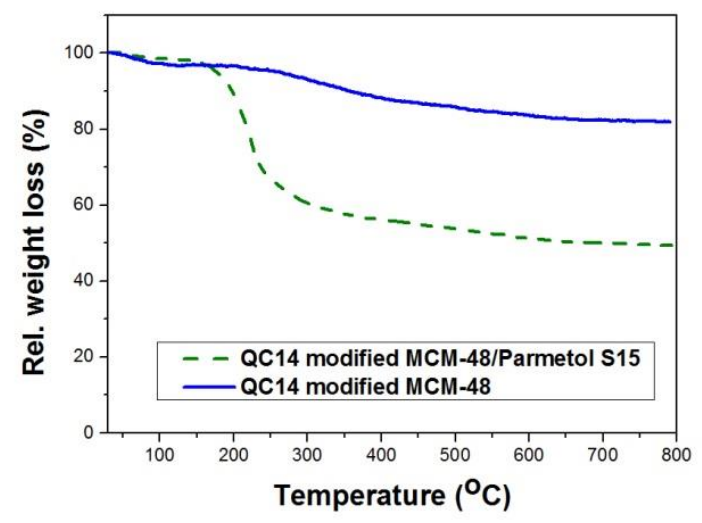

Figure 5. TGA curves for (a) the loaded/unloaded QC18-modified MCM-48 and (b) the loaded/unloaded QC14-modified MCM-48.

\section{E. coli}

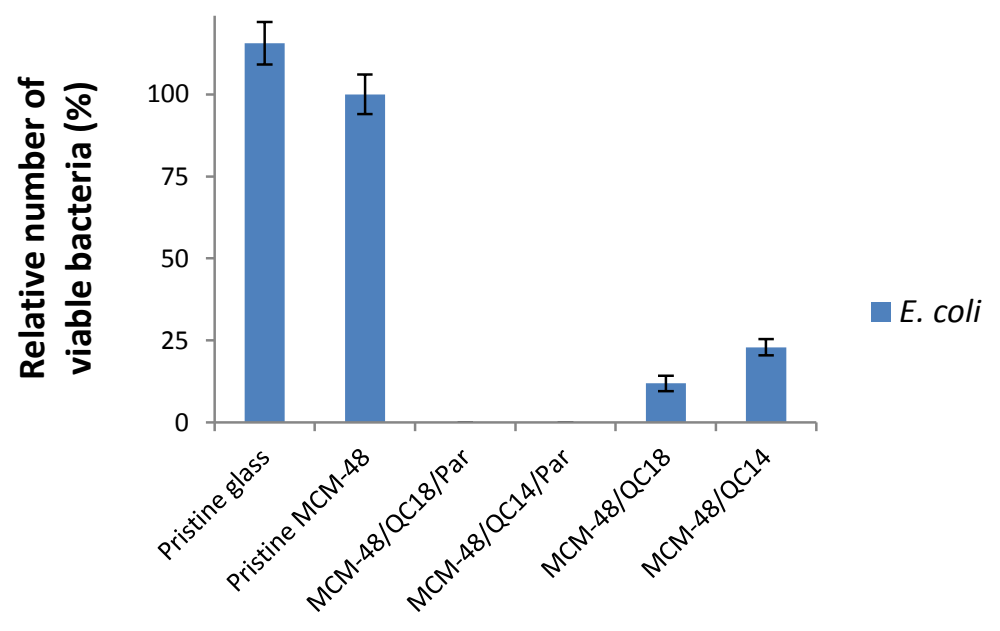

Figure 6. Relative number of viable bacteria (E. coli) after testing on glass slides spin-coated with pristine MCM-48, loaded/unloaded QC18 and QC14 modified MCM-48. Statistically significant differences between the samples $(p<0.0005)$. 


\section{S. aureus}

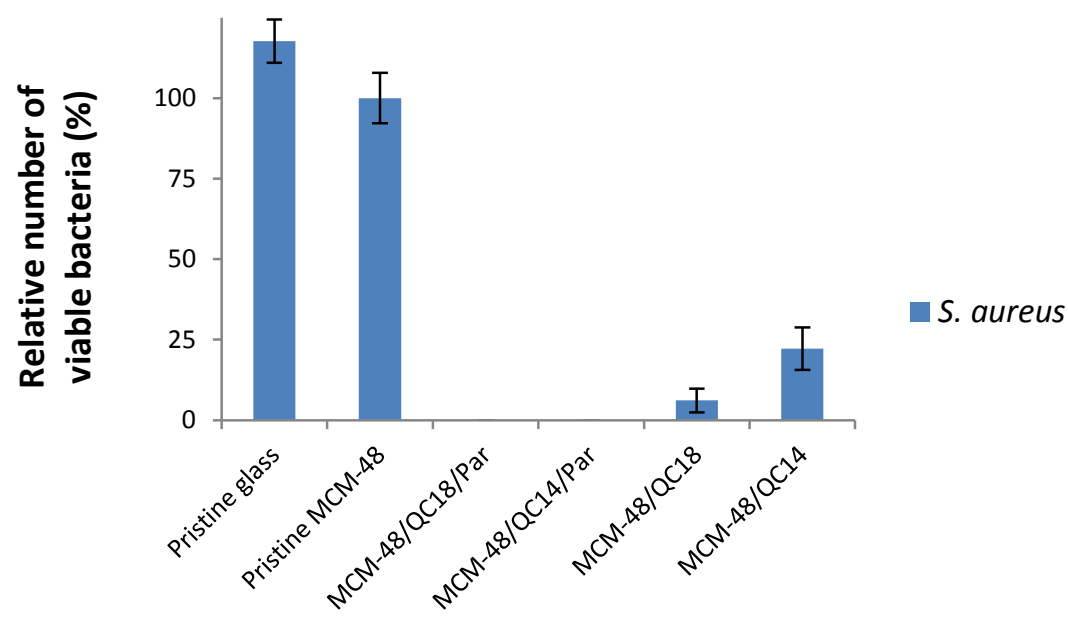

Figure 7. Relative number of viable bacteria (S. aureus) after testing on glass slides spin-coated with pristine MCM-48, loaded/unloaded QC18 and QC14 modified MCM-48. Statistically significant differences between the samples $(p<0.0005)$. 

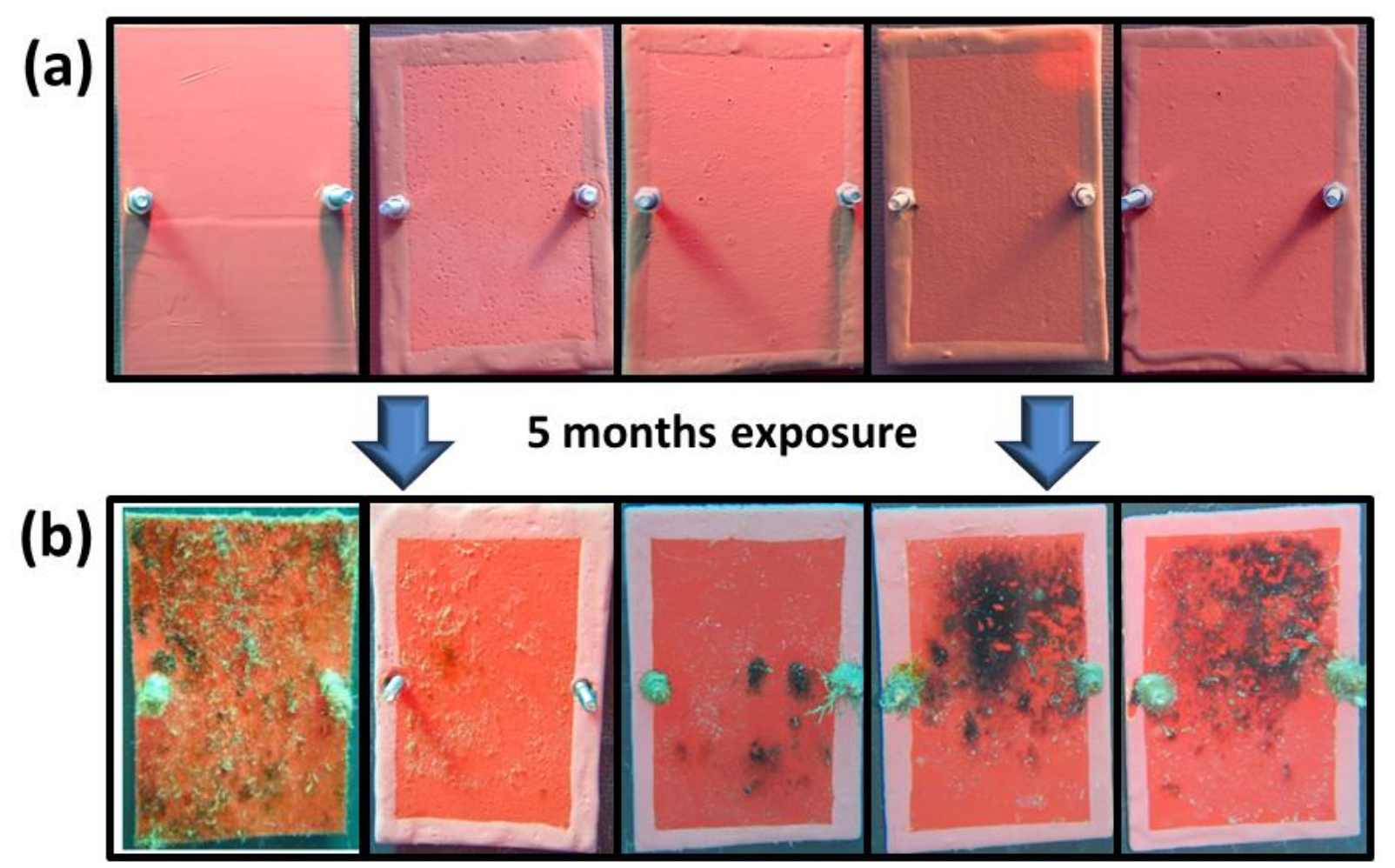

$\begin{array}{lllll}\text { Pristine Paint } & \text { Paint } 1 & \text { Paint } 2 & \text { Paint } 3 & \text { Paint } 4\end{array}$

Figure 8. Photographs of PVC panels coated with Pristine Paint and Paints 1-4 for the field test trial (Eilat, northern Red Sea) in the first day of deployment (a) and after 5 months of exposure (b).

Table 1. $\zeta$-potential measurements (right) and elemental analysis (left) for the pristine, QC18 and QC14 modified MCM-48.

\begin{tabular}{|cccc|}
\hline \multirow{2}{*}{ Sample } & \multicolumn{3}{c|}{$\begin{array}{c}\text { Elemental analysis } \\
(\text { wt } \%)\end{array}$} \\
\hline Pristine MCM-48 & 1.08 & 0.53 & 0 \\
\hline QC18-modified MCM-48 & 20.24 & 3.95 & 0.98 \\
\hline QC14-modified MCM-48 & 15.12 & 3.09 & 0.73 \\
\hline
\end{tabular}

\begin{tabular}{|cccc|}
\hline pH value & $\begin{array}{c}\text { Pristine } \\
\text { MCM-48 } \\
(\mathbf{m V})\end{array}$ & $\begin{array}{c}\text { QC18- } \\
\text { modified } \\
\text { MCM-48 } \\
(\mathbf{m V})\end{array}$ & $\begin{array}{c}\text { QC14- } \\
\text { modified } \\
\text { MCM-48 } \\
(\mathbf{m V})\end{array}$ \\
\hline $\mathbf{1 . 5}$ & $3.0 \pm 1$ & $32.5 \pm 2$ & $30.1 \pm 1$ \\
\hline $\mathbf{4}$ & $-31.0 \pm 2$ & $63.3 \pm 2$ & $49.2 \pm 1$ \\
\hline $\mathbf{7 . 5}$ & $-28.3 \pm 1$ & $52.8 \pm 1$ & $43.0 \pm 2$ \\
\hline $\mathbf{9 . 5}$ & $-33.9 \pm 1$ & $50.1 \pm 1$ & $35.8 \pm 1$ \\
\hline
\end{tabular}




\section{ASSOCIATED CONTENT}

\section{Supporting Information.}

The Supporting Information is available free of charge on the ACS Publications website.

Further information about the mechanism of surface modification, reaction of synthesis for the

QC14, ${ }^{1} \mathrm{HNMR}$ spectra and images for the synthesised materials (PDF).

\section{AUTHOR INFORMATION}

\section{Corresponding Authors}

*E-mail: Marios.Michailidis@liverpool.ac.uk

*E-mail: D.Shchukin@liverpool.ac.uk

\section{ORCID}

Marios Michailidis: 0000-0001-8845-2375

Dmitry Shchukin: 0000-0002-2936-804x

\section{Notes}

The authors declare no competing financial interest.

\section{ACKNOWLEDGMENT}

This work was financially supported by EU FP7 BYEFOULING project of EU Commission. We thank the Interuniversity Institute for Marine Sciences in Eilat (IUI) for assistance and use of facilities. We acknowledge M. Weis, and E. Gunter-Hoch for help in the field work. This 
research was in part supported by the Israel Cohen Chair in Environmental Zoology to YB. The field test complied with a permit issued by the Israel Nature and National Parks Protection Authority.

\section{REFERENCES}

(1) Callow, J. A.; Callow, M. E. Trends in the Development of Environmentally Friendly Fouling-Resistant Marine Coatings. Nat. Commun. 2011, 2, 244.

(2) Callow, M. E.; Callow, J. A. Marine Biofouling: A Sticky Problem. Biologist. 2002, pp $10-14$.

(3) Chambers, L. D.; Stokes, K. R.; Walsh, F. C.; Wood, R. J. K. Modern Approaches to Marine Antifouling Coatings. Surf. Coatings Technol. 2006, 201, 3642-3652.

(4) Magin, C. M.; Cooper, S. P.; Brennan, A. B. Non-Toxic Antifouling Strategies. Mater. Today 2010, 13 (4), 36-44.

(5) Schultz, M. P.; Bendick, J. A.; Holm, E. R.; Hertel, W. M. Economic Impact of Biofouling on a Naval Surface Ship. Biofouling 2011, 27 (1), 87-98.

(6) Fitridge, I.; Dempster, T.; Guenther, J.; de Nys, R. The Impact and Control of Biofouling in Marine Aquaculture: A Review. Biofouling 2012, 28 (7), 649-669.

(7) Yebra, D. M.; Kiil, S.; Dam-Johansen, K. Antifouling Technology - Past, Present and Future Steps towards Efficient and Environmentally Friendly Antifouling Coatings. Progress in Organic Coatings. 2004, pp 75-104. 
(8) Almeida, E.; Diamantino, T. C.; de Sousa, O. Marine Paints: The Particular Case of Antifouling Paints. Progress in Organic Coatings. 2007, pp 2-20.

(9) IMO. Focus on IMO: Anti-Fouling Systems. Int. Marit. Organ. 2002, 44 (January), 1-31.

(10) Adams, J. R.; Antoniadou, A.; Hunt, C. O.; Bennett, P.; Croudace, I. W.; Taylor, R. N.; Pearce, R. B.; Earl, G. P.; Flemming, N. C.; Moggeridge, J.; Whiteside, T.; Oliver, K.; Parker, A. J. The Belgammel Ram, a Hellenistic-Roman Bronze Proembolion Found off the Coast of Libya: Test Analysis of Function, Date and Metallurgy, with a Digital Reference Archive. Int. J. Naut. Archaeol. 2013, 42 (1), 60-75.

(11) Lejars, M.; Margaillan, A.; Bressy, C. Fouling Release Coatings: A Nontoxic Alternative to Biocidal Antifouling Coatings. Chem. Rev. 2012, 112 (8), 4347-4390.

(12) Gao, H.; Wen, D.; Sukhorukov, G. B. Composite Silica Nanoparticle/polyelectrolyte Microcapsules with Reduced Permeability and Enhanced Ultrasound Sensitivity. J. Mater. Chem. B 2015, 3 (9), 1888-1897.

(13) Zheng, Z.; Chang, Z.; Xu, G. K.; McBride, F.; Ho, A.; Zhuola, Z.; Michailidis, M.; Li, W.; Raval, R.; Akhtar, R.; Shchukin, D. Microencapsulated Phase Change Materials in SolarThermal Conversion Systems: Understanding Geometry-Dependent Heating Efficiency and System Reliability. ACS Nano 2017, 11 (1), 721-729.

(14) Zheng, Z.; Schenderlein, M.; Huang, X.; Brownbill, N. J.; Blanc, F.; Shchukin, D. Influence of Functionalization of Nanocontainers on Self-Healing Anticorrosive Coatings. ACS Appl. Mater. Interfaces 2015, 7 (41), 22756-22766.

(15) Gao, H.; Goriacheva, O. A.; Tarakina, N. V.; Sukhorukov, G. B. Intracellularly 
Biodegradable Polyelectrolyte/Silica Composite Microcapsules as Carriers for Small Molecules. ACS Appl. Mater. Interfaces 2016, 8 (15), 9651-9661.

(16) Shchukina, E. M.; Shchukin, D. G. LbL Coated Microcapsules for Delivering Lipid-Based Drugs. Adv. Drug Deliv. Rev. 2011, 63 (9), 837-846.

(17) Andersson Trojer, M.; Nordstierna, L.; Bergek, J.; Blanck, H.; Holmberg, K.; Nydén, M. Use of Microcapsules as Controlled Release Devices for Coatings. Adv. Colloid Interface Sci. 2015, 222, 18-43.

(18) Maia, F.; Silva, A. P.; Fernandes, S.; Cunha, A.; Almeida, A.; Tedim, J.; Zheludkevich, M. L.; Ferreira, M. G. S. Incorporation of Biocides in Nanocapsules for Protective Coatings Used in Maritime Applications. Chem. Eng. J. 2015, 270, 150-157.

(19) Sørensen, G.; Nielsen, A. L.; Pedersen, M. M.; Poulsen, S.; Nissen, H.; Poulsen, M.; Nygaard, S. D. Controlled Release of Biocide from Silica Microparticles in Wood Paint. Prog. Org. Coatings 2010, 68 (4), 299-306.

(20) Tischer, M.; Pradel, G.; Ohlsen, K.; Holzgrabe, U. Quaternary Ammonium Salts and Their Antimicrobial Potential: Targets or Nonspecific Interactions? ChemMedChem 2012, 7 (1), $22-31$.

(21) Xue, Y.; Xiao, H.; Zhang, Y. Antimicrobial Polymeric Materials with Quaternary Ammonium and Phosphonium Salts. Int. J. Mol. Sci. 2015, 16 (2), 3626-3655.

(22) Hegstad, K.; Langsrud, S.; Lunestad, B. T.; Scheie, A. A.; Sunde, M.; Yazdankhah, S. P. Does the Wide Use of Quaternary Ammonium Compounds Enhance the Selection and Spread of Antimicrobial Resistance and Thus Threaten Our Health? Microb. Drug Resist. 
2010, $16(2), 91-104$.

(23) Maillard, J. Y. Bacterial Target Sites for Biocide Action. Symp. Ser. Soc. Appl. Microbiol. 2002, No. 31, 16S-27S.

(24) Tashiro, T. Antibacterial and Bacterium Adsorbing Macromolecules. Macromol. Mater. Eng. 2001, 286 (2), 63-87.

(25) Davies, A.; Bentley, M.; Field, B. S. Comparison of the Action of Vantocil, Cetrimide and Chlorhexidine on Escherichia Coli and Its Spheroplasts and the Protoplasts of Gram Positive Bacteria. J. Appl. Bacteriol. 1968, 31 (4), 448-461.

(26) Gottenbos, B.; Van Der Mei, H. C.; Klatter, F.; Nieuwenhuis, P.; Busscher, H. J. In Vitro and in Vivo Antimicrobial Activity of Covalently Coupled Quaternary Ammonium Silane Coatings on Silicone Rubber. Biomaterials 2002, 23 (6), 1417-1423.

(27) Nurdin, N.; Helary, G.; Sauvet, G. Biocidal Polymers Active by Contact. II. Biological Evaluation of Polyurethane Coatings with Pendant Quaternary Ammonium Salts. J. Appl. Polym. Sci. 1993, 50 (4), 663-670.

(28) Saif, M. J.; Anwar, J.; Munawar, M. A. A Novel Application of Quaternary Ammonium Compounds as Antibacterial Hybrid Coating on Glass Surfaces. Langmuir 2009, 25 (1), $377-379$.

(29) Majumdar, P.; He, J.; Lee, E.; Kallam, A.; Gubbins, N.; Stafslien, S. J.; Daniels, J.; Chisholm, B. J. Antimicrobial Activity of Polysiloxane Coatings Containing Quaternary Ammonium-Functionalized Polyhedral Oligomeric Silsesquioxane. J. Coatings Technol. Res. 2010, 7 (4), 455-467. 
(30) Yoshihiro, N.; Hiroyuki, H.; Takahiko, T.; Hiroki, K.; Tokunaru, H.; Isao, S. Disinfection of Water with Quaternary Ammonium Salts Insolubilized on a Porous Disinfection of Water with Quaternary Ammonium Salts Insolubilized on a Porous Glass Surface. Appl. Environ. Microbiol. 1984, 47 (3), 513-518.

(31) Schumacher, K.; Grün, M.; Unger, K. . Novel Synthesis of Spherical MCM-48. Microporous and Mesoporous Materials. 1999, pp 201-206.

(32) Schumacher, K.; Ravikovitch, P. I.; Chesne, A. Du; Neimark, A. V; Unger, K. K. Characterization of MCM-48 Materials. Langmuir 2000, 16 (8), 4648-4654.

(33) Borodina, T. N.; Grigoriev, D. O.; Carillo, M. A.; Hartmann, J.; Moehwald, H.; Shchukin, D. G. Preparation of Multifunctional Polysaccharide Microcontainers for Lipophilic Bioactive Agents. ACS Appl. Mater. Interfaces 2014, 6 (9), 6570-6578.

(34) Jean Rouquerol, Françoise Rouquerol, Philip Llewellyn, Guillaume Maurin, K. S. Adsorption by Powders and Porous Solids. Acad. Press 2013.

(35) Brunauer, S.; Deming, L. S.; Deming, W. E.; Teller, E. On a Theory of the van Der Waals Adsorption of Gases. J. Am. Chem. Soc. 1940, 62 (7), 1723-1732.

(36) Romero, A.; Alba, M. D.; Zhou, W.; Klinowski, J. Synthesis and Characterization of the Mesoporous Silicate Molecular Sieve MCM-48. J. Phys. Chem. B 1997, 101 (27), 5294 5300.

(37) Kruk, M.; Jaroniec, M.; Ryoo, R.; Sang Hoon Joo. Characterization of MCM-48 Silicas with Tailored Pore Sizes Synthesized via a Highly Efficient Procedure. Chem. Mater. 2000, $12(5), 1414-1421$. 
(38) Kruk, M.; Jaroniec, M.; Ryoo, R.; Kim, J. M. Characterization of High-Quality MCM-48 and SBA-1 Mesoporous Silicas. Chem. Mater. 1999, No. 11, 2568-2572.

(39) J. C. Vartuli, K. D. Schmitt, C. T. Kresge, W. J. Roth, M. E. Leonowicz, S. B. McCullen, S. D. Hellring, J. S. Beck, J. L. Schlenker, D. H. Olson, E. W. S. Effect of Surfactant / Silica Molar Ratios on the Formation of Mesoporous Molecular Sieves : Inorganic. Chem. Mater. 1994, 6, 2317-2326.

(40) Brunauer, S.; Emmett, P. H.; Teller, E. Adsorption of Gases in Multimolecular Layers. $J$. Am. Chem. Soc. 1938, 60 (1), 309-319.

(41) Lastoskie, C.; Gubbins, K. E.; Quirke, N. Pore Size Distribution Analysis of Microporous Carbons: A Density Functional Theory Approach. J. Phys. Chem. 1993, 97 (18), 47864796.

(42) Landers, J.; Gor, G. Y.; Neimark, A. V. Density Functional Theory Methods for Characterization of Porous Materials. Colloids Surfaces A Physicochem. Eng. Asp. 2013, $437,3-32$.

(43) Ravikovitch, P. I.; Domhnaill, S. C. O.; Neimark, A. V.; Schueth, F.; Unger, K. K. Capillary Hysteresis in Nanopores: Theoretical and Experimental Studies of Nitrogen Adsorption on MCM-41. Langmuir 1995, 11 (12), 4765-4772.

(44) Ravikovitch, P. I.; Haller, G. L.; Neimark, A. V. Adsorption Characterization of Mesoporous Molecular Sieves. Mesoporous Mol. Sieves 1998, Proc. 1st Int. Symp. 1998, $117,77-84$.

(45) Neimark, A. V; Ravikovitch, P. I.; Grün, M.; Schüth, F.; Unger, K. K. Pore Size Analysis 
of MCM-41 Type Adsorbents by Means of Nitrogen and Argon Adsorption. J. Colloid Interface Sci. 1998, 207 (1), 159-169.

(46) Iler, R. K. The Chemistry of Silica: Solubility, Polymerization, Colloid and Surface Properties, and Biochemistry. Wiley 1979, New York, 897.

(47) Si-Han Wu, C.-Y. M. and H.-P. L. Synthesis of Mesoporous Silica Nanoparticles. Chem. Soc. Rev. 2013, No. 42, 3862.

(48) Shchukin, D. G.; Shchukina, E. Capsules with External Navigation and Triggered Release. Curr. Opin. Pharmacol. 2014, 18, 42-46.

(49) Zheng, Z.; Huang, X.; Schenderlein, M.; Borisova, D.; Cao, R.; Möhwald, H.; Shchukin, D. Self-Healing and Antifouling Multifunctional Coatings Based on $\mathrm{pH}$ and Sulfide Ion Sensitive Nanocontainers. Adv. Funct. Mater. 2013, 23 (26), 3307-3314.

(50) Kügler, R.; Bouloussa, O.; Rondelez, F. Evidence of a Charge-Density Threshold for Optimum Efficiency of Biocidal Cationic Surfaces. Microbiology 2005, 151 (5), 13411348.

(51) Chen, C. Z.; Beck-Tan, N. C.; Dhurjati, P.; van Dyk, T. K.; LaRossa, R. A.; Cooper, S. L. Quaternary Ammonium Functionalized Poly(propylene Imine) Dendrimers as Effective Antimicrobials: Structure-Activity Studies. Biomacromolecules 2000, 1 (3), 473-480.

(52) Majumdar, P.; Lee, E.; Gubbins, N.; Christianson, D. A.; Stafslien, S. J.; Daniels, J.; Vanderwal, L.; Bahr, J.; Chisholm, B. J. Combinatorial Materials Research Applied to the Development of New Surface Coatings XIII: An Investigation of Polysiloxane Antimicrobial Coatings Containing Tethered Quaternary Ammonium Salt Groups. J. 
Comb. Chem. 2009, 11, 1115-1127.

(53) Schneider, C. A.; Rasband, W. S.; Eliceiri, K. W. NIH Image to ImageJ: 25 Years of Image Analysis. Nat. Methods 2012, 9 (7), 671-675. 